\title{
Wilhelm Hoffmann
}

\section{Aus Anlass einer Neuerscheinung}

Wilhelm Hoffmann (1901-1986) war von 1945 an fast 25 Jahre lang Direktor der Württembergischen Landesbibliothek Stuttgart. Ein ursprünglich zu seinem 100. Geburtstag geplanter Sammelband zu Leben und Wirken Wilhelm Hoffmanns wird nun demnächst nach mehrjähriger Arbeit im Thorbecke verlag erscheinen.

Wilhelm Hoffmann war der Sohn des württembergischen Theologen, Prälaten und Hofpredigers Konrad Hoffmann (1867-1959). In Stuttgart besuchte er zunächst bis 1915 das Karls-Gymnasium, nach dem Abitur dann die Evangelischen Seminare in den Klöstern Maulbronn und Blaubeuren, bevor er ab 1919 Evangelische Theologie in Tübingen und Marburg studierte. 1923 legte er die erste theologische Dienstprüfung ab und wurde Studentischer Geschäftsführer der Tübinger Studentenhilfe, eine Tätigkeit, die er bis 1926 ausübte. Anschließend begann er sein zweites Studium der Fächer Geschichte und Pädagogik in Berlin und Tübingen, das er 1928 mit der Promotion abschloss. Von 1928 bis 1931 war Hoffmann dann Geschäftsführer und Leiter der Studienstiftung des Deutschen Volkes im Deutschen Studentenwerk in Dresden.

Um einen „Brotberuf“ zu erlernen, begann Hoffmann 1931 das Bibliotheksreferendariat an der Württembergischen Landesbibliothek in seiner Heimatstadt Stuttgart. Hier erhielt er nach Ablegung der Prüfung auch eine Stelle als Bibliotheksassessor. 1937 wurde er Bibliotheksrat. Zunächst war er Fachreferent für Theologie, Philosophie, Pädagogik sowie württembergische Geschichte, ab 1938 leitete er die Handschriften-Sammlung. Seit 1942 war Hoffmann zusätzlich zu seinen Aufgaben zeitweilig Leiter des neu gegründeten HölderlinArchivs. Im Zweiten Weltkrieg war Hoffmann sehr stark damit beschäftigt, die wertvollen Bestände der Württembergischen Landesbibliothek auszulagern, die so vor der Vernichtung bewahrt werden konnten.

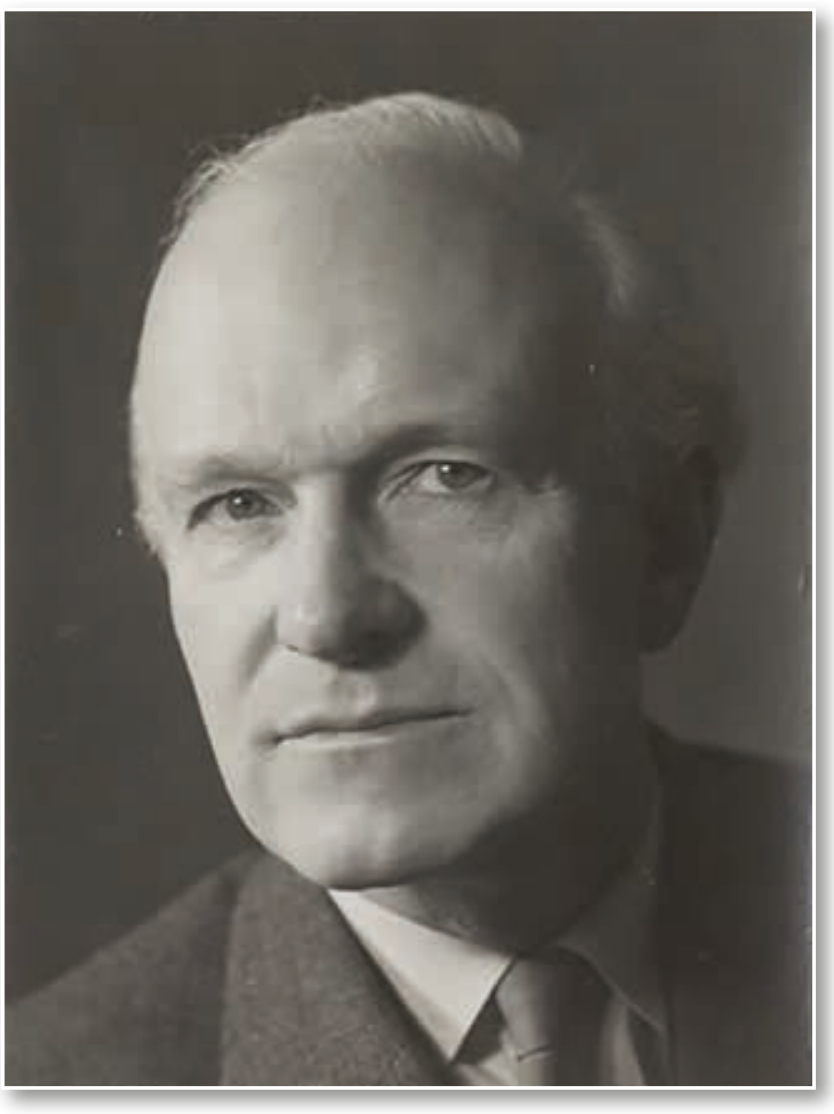

Nach dem Zweiten Weltkrieg wurde die alte, durch die NS-Zeit belastete Bibliotheksleitung abgesetzt, sodass Hoffmann nun Direktor der Württembergischen Landesbibliothek wurde. Er blieb dies fast 25 Jahre lang. Von 1946 bis 1951 war er zusätzlich kommissarischer Direktor der Universitätsbibliothek Tübingen.

Seine gesamte Amtszeit musste er in einem provisorischen Bibliotheksgebäude zubringen, das nur notdürftig in den wiederhergestellten Teilen des alten Bibliotheksgebäudes untergebracht war. Somit war diese Zeit auch sehr stark geprägt von der Suche nach baulichen Alternativen für die Bibliothek. Hoffmann favorisierte beispielsweise eine Unterbringung der Bibliothek im wiederaufzubauenden Neuen Schloss. Letztlich liefen die Planungen jedoch auf einen Neubau der Bibliothek hinaus, der aber erst nach Hoffmanns Amtszeit, im August 1970, eingeweiht werden konnte. Das Gebäude war jedoch ganz nach Hoffmanns Vor- 
stellungen geplant und ausgeführt worden. In Hoffmanns Amtszeit gelang auch der Wiederaufbau der Buchbestände, unter anderem konnte er reiche Spenden aus der Schweiz für die Bibliothek akquirieren. Die Bibliothek wurde durch Vorträge und Ausstellungen auch deutlich in das kulturelle Leben Stuttgarts eingebunden. Hier spiegelt sich Hoffmanns Auffassung wider, dass eine Bibliothek als Bildungsinstitution anzusehen sei. Reiche Förderung dieser Aktivitäten wurde der Bibliothek durch die Württembergische Bibliotheksgesellschaft zuteil, die von Wilhelm Hoffmann und anderen ins Leben gerufen wurde. Hoffmann konnte für die Gesellschaft bedeutende Förderer gewinnen: So war der württembergische Kult(us)minister und erste Bundespräsident Theodor Heuss in den ersten Jahren Schirmherr der Bibliotheksgesellschaft.

Um 1945 lernte Wilhelm Hoffmann Robert Boehringer (1884-1974) kennen, den Erben und Nachlassverwalter des deutschen Dichters Stefan George (1868-1933). Hoffmann wurde in den folgenden Jahren Gründungsvater der 1959 gegründeten Stefan George Stiftung und des Stefan George Archivs. Beide hatten ihren Sitz zunächst in Bebenhausen, seit 1970 befindet sich das Archiv im Gebäude der Württembergischen Landesbibliothek. Wilhelm Hoffmann betreute es von Beginn seines Ruhestandes bis zu seinem Tod 1986.

Seit 1948 arbeitete Hoffmann auch in der Deutschen Schillergesellschaft mit, als gewähltes Mitglied des Ausschusses der Gesellschaft, als Vizepräsident und von 1954 bis 1979 als Präsident. Schon zu Beginn seiner Amtszeit wurde die Idee geboren, in Marbach, dem Geburtsort von Friedrich Schiller, das Schiller-Nationalmuseum, das schon Anfang des 20. Jahrhunderts dort gegründet worden war, zu einem „Deutschen Literaturarchiv" auszubauen. 1955 wurde diese Einrichtung ganz offiziell aus der Taufe gehoben. Auch hier war Wilhelm Hoffmann wieder einer der Gründerväter.

Wilhelm Hoffmann erhielt für seine vielfältigen Tätigkeiten und Verdienste mehrere Ehrungen: So wurde er 1962 zum Ehrensenator der Universität Tübingen ernannt, 1978 erhielt er die Verdienstmedaille des Landes Baden-Württemberg und 1980 das Große Verdienstkreuz der Bundesrepublik Deutschland. In Stuttgart wurde an der Württembergischen Landesbibliothek ein Weg nach ihm benannt.

Wilhelm Hoffmann war verheiratet und hatte zwei Söhne: den Historiker Peter Hoffmann und den ehemaligen Oberbürgermeister von Biberach an der Riß, Claus-Wilhelm Hoffmann. Wilhelm Hoffmann verstarb 1986 vier Wochen vor Vollendung seines 85. Lebensjahres in Stuttgart.

Der nun erscheinende Sammelband enthält einen Beitrag von Claus-Wilhelm Hoffmann, der auch den Gesamtband herausgibt, über Wilhelm Hoffmanns Jahre bis 1930. Maria Gramlich widmet sich in ihrem Beitrag dem Wirken Wilhelm Hoffmanns in der Württembergischen Landesbibliothek von 1931 bis 1945 und wertet dabei in erster Linie die vielfältigen Materialien seines Nachlasses aus, den sie selbst erschlossen hat. Die beiden folgenden Aufsätze von Ute Oelmann, der langjährigen Leiterin des Stefan George Archivs, befassen sich mit Hoffmanns Nachkriegsjahren, unter anderem seinem Plan, in Bebenhausen ein „weltliches Kloster" zu errichten. Das Wirken Wilhelm Hoffmanns als spiritus rector der Württembergischen Bibliotheksgesellschaft beleuchtet der ehemalige Verleger Wulf-Dietrich von Lucius, der selbst jahrzehntelang Vorsitzender dieses Freundeskreises der Landesbibliothek war. Die drei abschließenden Beiträge behandeln die verschiedenen Archive, die Wilhelm Hoffmann (mit)gegründet hat: Zum einen das Hölderlin-Archiv, dessen Gründung und Geschichte bis 1960 Liselotte Lohrer beschreibt. Dies ist ein Beitrag von 1960, der hier wieder abgedruckt wird. Die Gründung der Stefan George Stiftung und des Stefan George Archivs wird von Ute Oelmann skizziert, während Ulrich Ott, der ehemalige Leiter des Deutschen Literaturarchivs, die Verbindung Wilhelm Hoffmanns mit dieser Einrichtung beleuchtet.

Hans-Christian Pust 